\title{
Narrative Approaches to Design Multi-screen Augmented Reality Experiences
}

\author{
Roma Patel \\ Nottingham Trent University \\ School of Art and Design \\ Burton Street, Nottingham NG1 4BU \\ United Kingdom \\ roma.patel@ntu.ac.uk
}

\author{
Deborah Tuck \\ Nottingham Trent University \\ School of Art and Design \\ Burton Street, Nottingham NG1 4BU \\ United Kingdom \\ deborah.tuck@ntu.ac.uk
}

\begin{abstract}
Augmented Reality (AR) represents the future of the digital integrated museum experience. There is considerable scope for providing engaging and interactive experiences when using AR combined with traditional museum practices, particularly relative to interpretive narrative. The new relationship created between the 'real' and 'virtual' object, generates new and engaging experiences and encourage more active visitor participation. AR can simultaneously layer competing accounts of historical events beyond the single voice of 'authority'.
\end{abstract}

This paper discusses the challenges of adapting and developing the first-hand witness accounts for a multi-screen AR experience of the night Nottingham Castle was attacked during the Reform Bill Riots (1831.) In each case we will present examples demonstrating our interdisciplinary design approach and the strategies we implemented for each story. Unlike many AR projects this approach places storytelling at its heart by integrating traditional theatrical and cinematic narrative techniques to create added suspense and engagement. It has opened up possibilities for learning and experiencing the site's history within a new interactive context, whilst simultaneously foregrounding the broader socio-political context around protest and riot. The exhibition will open to the public from May 2014 and the digital augmented environments will be fully integrated within the museum exhibition offering visitors a richer narrative with content generated through AR.

Riot 1831@ Nottingham Castle is supported by the Digital R\&D Fund for the Arts - Nesta, Arts \& Humanities Research Council and public funding by the National Lottery through Arts Council England.

\section{Augmented reality. Storytelling. Museum. Theatre. Narrative.}

\section{INTRODUCTION}

Nottingham Castle was established as the first municipal museum and art gallery outside London In 1875. A castle site since the 11 th Century, it has a chequered history of murders, sieges, and intrigue. The medieval castle was destroyed during the Civil War (1642-1651) and replaced by a Ducal Palace in 1674. This palace was then set alight by rioters on an October night in 1831. That night left a legacy, a unique combination of diverse first-hand witness accounts and museum objects and the Riot 1831@ Nottingham Castle' project aims to bring this history to life, using Augmented Reality (AR).

The research investigates the ways AR can add to the interpretive understanding of a heritage site and create opportunities to deepen engagement and empathy through multiple narrative perspectives. It uses $A R$ as an active storytelling medium to develop an understanding of the new relationships that can be formed between the 'real' and 'virtual' objects. The digital interpretation is developed alongside the redesign of a new exhibition to bring a contemporary interpretation to the historical collection. The project will result in a cross platform AR App that uses image recognition to augment real time $3 \mathrm{D}$ realtime computer environments, animated first-person performances and sounds onto museum objects, allowing the visitors to simultaneously interact and experience the events of 1831 at the exhibition.

This paper will first examine the role of storytelling and $A R$ in museums and give a brief overview of recent projects. It will then go on to focus on the narrative development and unique features of $A R$ for storytelling and discuss the influence of film and theatre practice in the digital interpretation and the challenges of adapting and developing the augmented story experience for an exhibition. 


\section{NARRATIVE AND AR IN MUSEUMS}

The art of storytelling has been a fundamental means of communicating throughout our existence and has long been recognised and used by museums and heritage sites. It is not uncommon to see the characters (costumed actors), roaming the halls of heritage sites evoking the past. Stories about heritage objects can add depth to knowledge as well as entertain allowing both the tangible and intangible to be explored simultaneously (Johnsson 2006). 'A story is different (from information). It does not expend itself. It preserves and concentrates its strength and is capable of releasing it even after a long time' (Benjamin 2000).

The problem is that though the storytelling is seen as beneficial in museums, when digital technologies have been used it tends to be in the form of information and data about the history or object, this is new, exciting and scaffolds their learning objectives but still the relationship to the artefact or place remains objective and distant. Storytelling can encourage a more subjective and emotional connection, assisting the visitor to feel empathy with history and characters. Several digital storytelling projects have been undertaken in museums to make exhibitions more engaging and accessible. However, there is a tension between the requirements for pedagogical outcomes and making the heritage more impressive, though virtual and augmented reality technologies (Kenderdine 2010). This tension somewhat eases, when the interpretation takes the form of a quest or a game or interactive digital storytelling.

'The Interactive Storytelling Exhibition Project' was based around Egyptian History and used kiosks and RFID tags. The results of this study found the game format was familiar to visitors and children were almost unanimous in saying that they enjoyed the exhibition and wanted to find out more. (Danks et al. 2006).

The age of mobile computing and applications (apps) has brought a multitude of digital options, for both the museum sector, as well as the visitor with the increasing use of tablets and smart phones, the technology has now become more accessible. The Museum Associations' (MA) mobile survey confirms that the sector is fully aware of the advantages of using mobile technologies. The results found that most of their respondents believed that QR codes were appropriate to use in cultural sectors and over half $(58 \%)$ felt it was essential for visitor engagement ( MA 2012). Many museums prefer visitors to bring their own device, the implication of this is that it would exclude some visitors form access the exhibition fully. Augmented reality can be one of the more challenging mobile technologies for museums to implement, but it is also potentially the most ideal current digital format to use for the museum sector.

With the recent prevalence of object oriented recognition systems as opposed to the use of QR codes, the visitors' attention can now be more firmly focused on the museum object as the objects themselves, become the markers that triggers the digital experience. The 'live' digital experience can be overlaid and attached to objects at the control and demand of the visitor, the digital work is movable and the visitor can also gaze at, contemplate the object alone in the space, without any intruding labels, screens or wires, (McKinley \& Damala 2013). AR also has the flexibility to allow museums to look beyond their wall, with the use of GPS the streets become a potential site for engagement with the Museum of London, AR app 'Street Museum' being one of the more successful examples of a type of site specific exhibition.

The British museum learning team has actively researched and implemented AR since 2011. They provided the tablets for their visitors and worksheets alongside specific exhibitions. 'Passport to the Afterlife' took the form of a family trail using QR code, while the more recently launched of 'Gift for Athena' (2013), an object recognition based AR trail and puzzle. Their first studies, they found that the live camera view seems to engage their visitors the most. 'AR is an inherently engaging interaction style. It's magical. Kids like it. Grown ups like it.' (Mannion 2012).

Unlike a game, storytelling does not offer the opportunity for the audience to win, it offers the opportunity for them to understand and feel human experiences. Stories engage us through our "vicarious eye" and "visceral eye", (Boorstin 1990) evoking emotions, empathy and sympathy for the character. To experience empathy the visitor needs to get to know the characters, understand their back story, their relationship to other people in the story, etc. This leads to them "caring" about what happens next, keeping them "glued" in anticipation. If AR storytelling is to capture the 'live' and immediacy of storytelling, it needs to be dramatised, go beyond the factual information found in many museums today. The dramatic interpretation can allow the museum visitor to be connected with, 'a knowledge that is felt than rationally understood' (Witcomb 2010). However, this poses a predicament for Museums as they are seen a the 'voice of authority', they need to get the right balance between the authenticity of the historical information and the need to tell an entertaining story (loannidis et al. 2012). 
Although there are many examples of $A R$ in museums, there are only few that exploit the benefits of storytelling using the dynamic and immersive elements of the medium. The 'live' nature of AR needs to be carefully considered in curating and interpreting the digital content. The augmented digital layers can become a montage to extend the spatial narrative changing the visitor's perception of the space and history.

The intentions of this project is not to replicate realism of a virtual reconstruction nor is it a virtual reenactment of 1831 Riots but its aim is to create a dramatisation of the eyewitness accounts of the events of that night designed to help the visitor "feel connected" to the people of the past. This approach was sometimes viewed with suspicion by historians and museum staff, consequently the creative team paid careful attention to each interpretation and consulted with historians and curators throughout the project. This helped to maintain a respective balance between historical fact and historical interpretations.

\section{THE NARRATIVE DEVELOPMENT}

The museum visitor traversing the virtual storyworld moving between multiple screens with multiple meaning- entering the space of the real objects - picking up fragments - making a connection-engaging with the past- each historic character with a story to tell of that eventful night in 1831 - leaving the visitor to fuse together their stories- making meaning of the history - confronted with today and yesterday - roots of the present and past...

The exhibition space becomes the 'storyworld', it is the juxtapostioning of AR episodes or little stories, the museum object and the site. Although there is a chronological journey, the order of the visitors' experience cannot be controlled, since the exhibition space has three different entrances. The visitors' curiosity will have to lead their exploration and interpretation. Research shows that people have a desire to know how a story end and two out of three people will do this at the expense of interactivity (Ryan 2001). What would happens if the visitor starts at the end? Would they try and seek out the linear route? How does this contribute to their experience of the story? How long should the AR experience be? These are the questions we have been grappling with when adapting the stories. Each experience needs to be designed to be viewed independently, but also make sense together. The context in which the experience is viewed is also significant, a visitor using their smart phone will have a different experience to the visitor using the ipad provided in the exhibition.

The curatorial relationship between the digital and physical exhibition are carefully considered, to create intermingling of the virtual and the real objects, the past and the present, it's not possible to view one without the other.

Making the AR experience accessible to all visitors was an essential part of this project, iPads are installed in the exhibition space, the app is cross platform and free to download and $\mathrm{Wi}-\mathrm{Fi}$ is provided in the gallery space.

\subsection{Designing the Storyworld}

The first phase of the project involved identifying the specific objects from the museum collection that would be used as AR markers to activate the stories (Fig 1). The choice was significant as the objects needed to firstly, relate to the historical eyewitness accounts and secondly, have a form that was can be 'tracked', tracking works out where a mobile device camera is looking so that AR graphics can be drawn in the correct place. The AR object recognition systems work (fig.2) well with High contrast image and forms and functions less

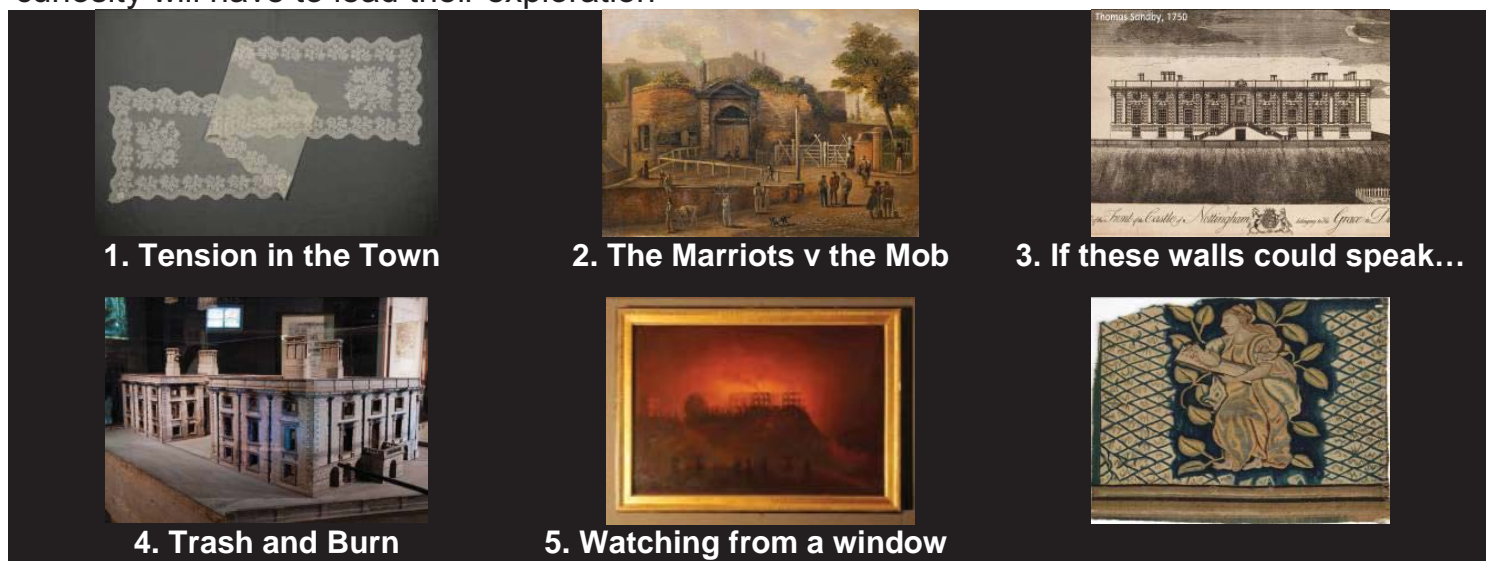

Figure 1: Five stories and six museum objects used for for the AR experience 
Efficiently with low contrasting images. In addition High contrast image and forms and functions less efficiently with low contrasting images. In addition the historic objects require protection from excessive light, restricting where they could be placed within the gallery and providing further complications for the exhibition design. This significantly restricted the range of museum objects we could use from the few we had.

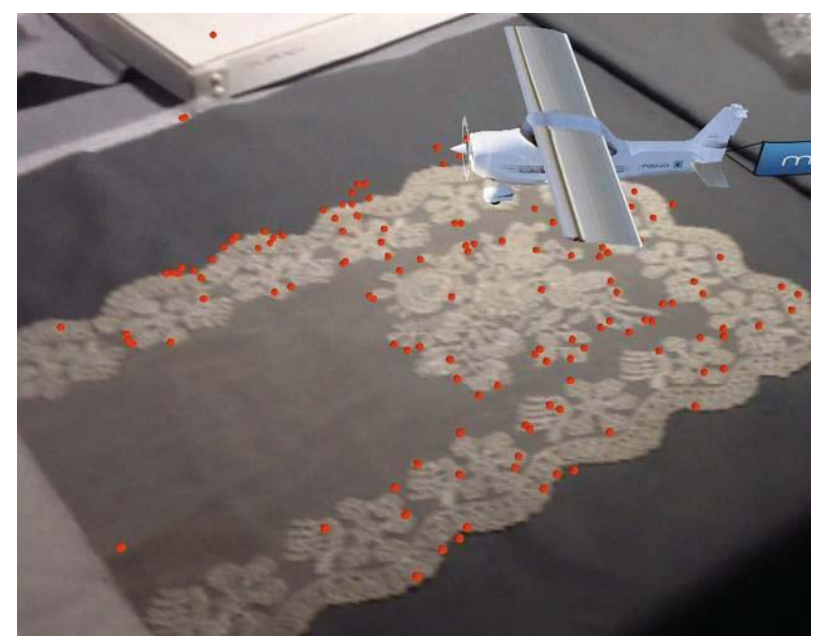

Figure 2: AR Tracking test of the lace scarf

The main interdisciplinary team was made up of academics and practitioners with experience in scenography, digital media, history, animation, exhibition design and film making. The first phase of the project involved the design and development of the narrative content Art and design researchers worked closely with the staff at the Nottingham Castle to adapt the existing eyewitness accounts of the riots into an engaging $A R$ experiences that span across five screens. Hot Knife Digital Media Ltd, the technology partner also played a key role as a consultant during this first phase of the project.

Working with a visual medium like AR allows for the exploration of non language based approaches in which 'an agent relates (tells) a story in a particular medium, such as language, imagery, sound, building, or a combination..' (Bal 1997). The relationship between the technology and the narrative became a significant throughout the design practice. A significant part of the process involves asking the question, when does this technology enhance the narrative and when does it not serve this purpose well?

The design, development included the use of scripts, storyboards and iterative prototyping. First and foremost when designing an AR narrative experience the relationship between the real object, the interactor (visitor) and the virtual image space must always be considered, the experience is more like designing for three dimensional space such as a site-specific performance or an installation rather than a screen based 2D animation. Thinking about all these elements was challenging, some of our initial approaches to the projection of content onto the artefacts did not take full advantage of the AR technology. The 'storyspace' was centred only onto the object's surface, once 'the user experience was enacted and discussions with our technology partners the virtual content was expanded further into the museum gallery space itself. For example, in the case of a wall mounted painting - the augmentation can 'brings the painting to life' similar to a Trompe l'oeil effect, but also beyond the frame, extending the virtual performance into the Gallery space, putting the visitor closer to the story. Augmenting the stories in the gallery space brought additional challenges, however, this exploration led to interpretations that are now more engaged and playful.

\subsubsection{Panoramas - The body in movement}

This spatial relationship is a unique element of $A R$ and can be used to great effect to immerse the viewer. The 360 degree realtime augmentation of the visual image in real space can be compared to the Panoramas of the 18th century, where the viewer is surrounded in an unbroken view of their surroundings. However, in AR the viewer's field of vision is restricted by the dimensions of the screen and the extent of the virtual projections, into the space can only be seen within this frame (screen). The visitor controls their view of the 'panorama by moving the frame around the space as they look.

This is explored by Moonbot's app 'The Fantastic Flying Books of Morris Lessmore' the 360 degree space reveals a magical imaginary world of the protagonist whilst the artist, Peter Weibel's AR installation, 'Life in the 20th Century, 225 Million Murders' facts about deaths, caused by genocides, wars and murders is used to confront the viewer directly, bringing difficult subjects into her real environment rather than in some distant place. Both the apps use the same technique to elicit completely different reactions.

The virtual image space becomes a scenographic space, where the arrangement of the narrative layers becomes dynamic. 'Space becomes an 'acting place' rather than a place of action.' (Bal 1997 pp136). Similarly, the strength of site- specific theatre is the extent in which the performance becomes inseparable from the narrative of the site, and in the case of heritage and history, the past and the present can be merged. (Pearson 2011). Adopting a site specific method of practice was particularly useful when interpreting two of the episodes which made use of panoramic AR. 


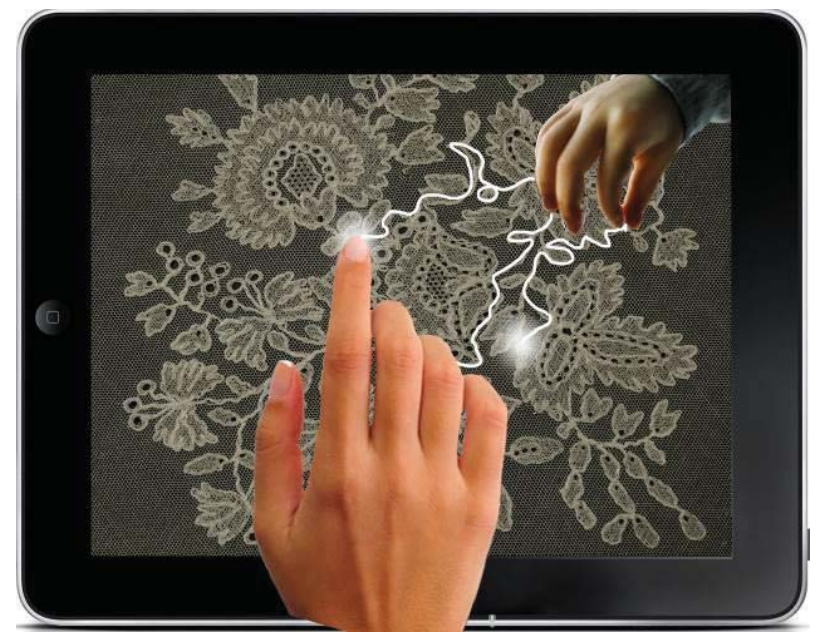

Figure 3: Storyboard of a visitor helping John

In "Tension in the Town" the visitor approaches a display containing a lace scarf, fixed and hanging in front is an iPad device. She adjusts the screen view to point over the lace display and an animation of a close up view of the hand of a young boy appears onto the screen. (fig 3 ) The hand begins unpicking a lace scarf; the boy is helping his mother to fix mistakes, he talks to the visitor. He asks the visitor to help him unravel the scarf. Then, as the visitor touches the scarf to help the lace threads animate and float into the gallery space (virtual vision), they then morph into words as they float.

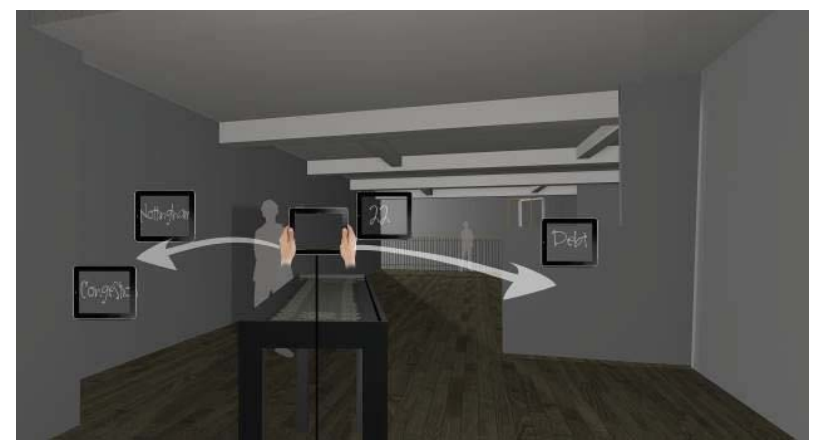

Figure 4: $3 D$ visualisation of the interaction for the lace words panoramic scene

The visitor is then prompted, to move the iPad up and around the space to see panoramic scenes of 'lacewords', highlighting the poverty stricken state of Nottingham's poor in 1831. (fig. 4) The virtual strings of lace float around the space and the gallery becomes the location for the performance. As this lace fields floats over the scale model of the town and other museum objects, the sounds of people from the past, whispering their words of poverty are heard. The intention is for the visitor to make connections between the visualisation and the museum objects as well as encouraging them to dwell, play and explore.

\subsubsection{Character's journey}

Exposition is used within storytelling as a means to introduce a character to the audience and help them get to know them better by providing essential contextual and background information that informs them of the character they have met. Each AR episode is experienced on different screen around the gallery, in order to connect and cohere the stories, the character development and exposition was created over several different episodes, by reintroducing the character 'John' the boy who died in the fire within three of the five episodes. Once as the boy unravelling the scarf, another as a shadow in the Ducal palace as it burned and he can also be seen as a party to the 'mob who stormed the castle. This allows the visitor to become familiar with the character and begin to develop an understanding and relationship to them, assisting their ability to empathise with the story. The aim is when visitors move through the physical gallery space, they are in fact moving in the 'storyworld' and hopefully maintaining a memory of the character.

John's story is made up of a variety of factual fragments about a young boy who died in the fire, we have pieced these together interpreting them to present the plight of poor children who lived in Nottingham at that time. There are so few facts or artefacts left of the riots, a handful of eyewitness accounts tells the story and so again, interpreting these stories is essential, to fill in the gaps of knowledge provided by directly related evidence. We fill these gaps, between the facts, with appropriately considered content, created in consultation with specialist historians and academics.

\section{FOCUS GROUP}

The research and development process for project includes four focus group meetings that will be conducted for the purpose of refining the content, design and interactive features of the AR app and the exhibition space design. The first focus group took place two months into the project with eight participants. They were taken on a physical "mock up" of the exhibition, the narrative content and AR interactions were demonstrated using storyboards and they also had hands-on experience of an early version of the AR prototype, using a handheld device.

The dominant perception was that the narratives were strong and helped to reinforce the theme of Rioting, in particular participants felt that some of the stories inspired them to find out more about a particular character, such as the young boy. While all agreed on the benefits of using AR in the exhibition and its potential to be engaging, one 
participant felt we need to be careful that some of the narrative interpretations do not appear overly focus on children and that there is a good balance for both the child and adult visitor.

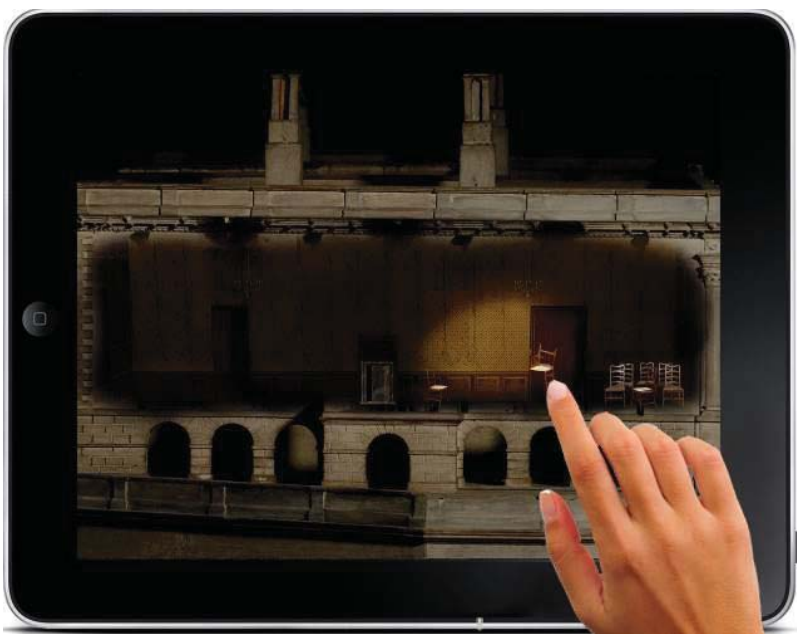

Figure 5: Storyboard of a visitor, helping stack chairs

The discussions about posing questions to the visitor, such as voting or helping the rioters burn the Castle highlighted that these can be made more effective and requires further consideration, (fig. 5) i.e. what points in the exhibition and the App will these be embedded and how will they complement each other. The group suggested that asking the audience to interact with the display, by helping rioters to stack and burn chairs in the Gallery put the audience into a difficult position, asking them to take part in the riots could be seen as offensive and provocative. This was in response once the focus group was aware of the modern day parallels being made between the riots of yesterday and those of today. By seeing a mobile phone within the augmented animated story, connections to the present were made.

This may put the visitor in a challenging position about whether to take part in the story. Initial observations of the focus group using AR indicated a certain amount of interaction and time is needed to allow the visitor to become used to the technology, after the amazement of seeing it for the first time. The participants interacted more with each other, observed each other, when using the application and this created a friendly and "safe" environment for their discovery.

\section{CONCLUSIONS AND FUTURE WORK}

This project was undertaken to investigate how storytelling can be incorporated into AR. The findings have enhanced our understanding of the medium and go some way towards demonstrating several practical applications of using AR to enhance storytelling in a museum setting.
However, although our methods and interdisciplinary approach to the subject was essential to the development of this project, it can be time consuming and not always practical for smaller museums to implement.

This project is in its second phase. The major narrative development is completed and our technology partner is currently building and developing the AR episodes and app. There is an agile approach to this phase of the development. Testing takes place weekly with the partners in order to make quick changes, adapting to the knowledge gained as our understanding of the medium, possibilities and limitations grow. In order to evaluate the narrative approaches discussed in this paper, considerably more work has to be done when the exhibition opens. There will be a six week period where qualitative and quantitative research will be undertaken, including eye tracking and video ethnography with the gallery visitors and smaller focus groups.

\section{REFERENCES}

Bal, M. (1997) Narratology, Introduction to the Theory of Narrative. University of Toronto Press, Toronto.

Benjamin, W. (2000) The Storyteller: In McKeon, M. (ed.), Theory of the Novel: A Historical Approach. John Hopkins Univesity Press.

Boorstin, J. (1995) Making Movies Work: Thinking Like a Filmmaker. Silman-James Press, Los Angeles.

Danks, M., Goodchild, M., Rodriguez-Echavarria, K., Arnold, D B., and Griffiths, R. (2007) Interactive Storytelling and Gaming Environments for Museums: The Interactive Storytelling Exhibition Project Technologies for E-Learning and Digital Entertainment, pp. 104-115.

Ioannidis, Y., El Raheb, K., Toli, E., Boile, M., Katifori, A., and Mazura, M. (2013) One object many stories: Introducing ICT in museums and collections through digital storytelling. In Digital Heritage International Congress 2013, Marseille, France, pp. 421-424. IEEE.

Johnsson, E. (2006) Telling Tales: A guide to developing effective storytelling programmes for museums. Renaissance London. C. Adler (ed.). http://www.museumoflondon.org.uk/files/4413/7468 13728/Telling-Tales.pdf (retrieved 16 Feburary 2014).

Kenderdine, S. (2010) Speaking in Rama: Panoramic vision in cultural heritage visualization: In Cameron, F and Kenderdine, S. (eds.), Theorizing Digital Cultural Heritage, The MIT Press, Cambridge, MA. 
Mannion, S. ( 2012) Beyond cool: making mobile augmented reality work for museum. In MW2012: Museum and the Web 2012, San Diego, USA. http://www.museumsandtheweb.com/mw2012/pap ers/beyond cool making mobile augmented reali ty wo.html (retrieved 10 January 2013).

McKinley, R., and Damala, A. (2013) ARtSENSE and Manifest. AR: Revisiting museums in the public realm through emerging art practices. In MW2013: Museums and the Web 2013, Portland, USA. http://mw2013.museumsandtheweb.com/paper/arts ense-and-manifest-ar-revisiting-museums-in-thepublic-realm-through-emerging-art-practices/ (retrieved 18 May 2014).
Museum Association Mobile survey (2012) Museum Association, http://www.museumsassociation.org/download?id= 731198 (retrieved 20 Feburary 2013)

Pearson, M. (2010) Site Specfic Performance, Palgrave, Macmillan, UK.

Ryan, M (2001) Narrative as Virtual Reality. John Hopkins Univesity Press. Baltimore and London.

Witcomb, A. F. (2010) The Materiality of the Virtual Thechnologies. A New Approach to Thinking about the impact of Multimedia In Museums In Cameron, F., and Kenderdine, S. (eds.), Theorizing Digital Cultural Heritage. The MIT Press, Cambridge, MA. 